\title{
Manipulation under k-approval scoring rules
}

Citation for published version (APA):

Peters, H. J. M., Roy, S., \& Storcken, A. J. A. (2008). Manipulation under k-approval scoring rules. METEOR, Maastricht University School of Business and Economics. METEOR Research Memorandum No. 056 https://doi.org/10.26481/umamet.2008056

Document status and date:

Published: 01/01/2008

DOI:

10.26481/umamet.2008056

Document Version:

Publisher's PDF, also known as Version of record

\section{Please check the document version of this publication:}

- A submitted manuscript is the version of the article upon submission and before peer-review. There can be important differences between the submitted version and the official published version of record.

People interested in the research are advised to contact the author for the final version of the publication, or visit the DOI to the publisher's website.

- The final author version and the galley proof are versions of the publication after peer review.

- The final published version features the final layout of the paper including the volume, issue and page numbers.

Link to publication

\footnotetext{
General rights rights.

- You may freely distribute the URL identifying the publication in the public portal. please follow below link for the End User Agreement:

www.umlib.nl/taverne-license

Take down policy

If you believe that this document breaches copyright please contact us at:

repository@maastrichtuniversity.nl

providing details and we will investigate your claim.
}

Copyright and moral rights for the publications made accessible in the public portal are retained by the authors and/or other copyright owners and it is a condition of accessing publications that users recognise and abide by the legal requirements associated with these

- Users may download and print one copy of any publication from the public portal for the purpose of private study or research.

- You may not further distribute the material or use it for any profit-making activity or commercial gain

If the publication is distributed under the terms of Article $25 \mathrm{fa}$ of the Dutch Copyright Act, indicated by the "Taverne" license above, 
Hans Peters, Souvik Roy, Ton Storcken

Manipulation under $k$-approval scoring rules

$\mathrm{RM} / 08 / 056$

JEL code: D71, D72

\section{METEबrR}

Maastricht research school of Economics of TEchnology and ORganizations

Universiteit Maastricht

Faculty of Economics and Business Administration P.O. Box 616

NL - 6200 MD Maastricht

phone : :++31 433883830

fax : :+31433884873 


\title{
Manipulation under $k$-approval scoring rules
}

\author{
Hans Peters* Souvik Roy* Ton Storcken*
}

This version, December 2008

\begin{abstract}
Under a $k$-approval scoring rule each agent attaches a score of one to his $k$ most preferred alternatives and zero to the other alternatives. The rule assigns the set of alternatives with maximal score. Agents may extend preferences to sets in several ways: they may compare the worst alternatives, or the best alternatives, or use a stochastic dominance criterion. In this paper we characterize the non-manipulable profiles for each of these set comparisons. For two-agent profiles we also determine the value(s) of $k$ for which the number of non-manipulable profiles is maximal.
\end{abstract}

JEL-Classification: D71, D72

Keywords: $k$-approval scoring rules, (non-)manipulability

*Department of Quantitative Economics, University of Maastricht, PO Box 616, 6200 MD Maastricht, The Netherlands. E-mail addresses: h.peters@ke.unimaas.nl, s.roy@ke.unimaas.nl, t.storcken@ke.unimaas.nl. 


\section{Introduction}

In most voting situations agents have the possibility to manipulate the outcome of the vote by not voting according to their true preferences. The classical theorem of Gibbard (1973) and Satterthwaite (1975) formalizes this fact for social choice functions with unique outcomes. The present paper belongs to the strand of literature, initiated by Kelly $(1988,1989)$, which accepts this phenomenon as a matter of fact and looks for social choice rules which are second best in this respect, i.e., least manipulable. ${ }^{1}$ Specifically, we consider a variation on a well-known and often used voting procedure, approval voting (Brams and Fishburn, 1983). In approval voting each agent can approve of as many alternatives as he wants. It is well known that approval voting is strategy-proof (non-manipulable) if preferences are dichotomous that is, each agent distinguishes between a set of good and a set of bad alternatives. To avoid this restriction and allow for strict preferences (linear orders) we consider $k$-approval voting, where each agent approves of exactly $k$ alternatives. In a companion paper (Peters et al. 2009) we consider non-manipulability of approval voting (without fixed $k$ ) under several assumptions on preferences, intermediate between strict preferences and dichotomous preferences.

We assume that agents have strict preferences over alternatives. Since $k$ approval voting may result in a set of outcomes rather than a single outcome, we consider several preference extensions to sets: comparing sets by their worst alternatives; by their best alternatives; and by stochastic dominance, assuming each alternative in a set to be equally likely. We then characterize the preference profiles at which the $k$-approval scoring rule is non-manipulable.

An important goal of this effort is to find the value of $k$ for which the number of non-manipulable preference profiles is maximal, i.e., for which $k$-approval voting is least manipulable. So far we have managed to obtain theoretical results only for the case of two agents. For $k=1, k$-approval voting is just plurality voting and this is non-manipulable (strategy-proof) for two agents under all three preference extensions. Plurality voting, however, has a serious drawback. If (the) two agents agree on a good second-ranked alternative but disagree on the first, then under plurality voting this compromise is not chosen; it would be chosen, however, under any other $k$-approval scoring rule. Therefore, for each of the three mentioned preference comparisons and for $k \neq 1$ we have established the overall optimal value of $k$, and the optimal value under the restriction $k \leq m / 2$, where $m$ is the total the number of alternatives. The latter restriction is justified by the desirable property of 'citizen sovereignty': for each alternative there is a preference profile resulting in that alternative as the unique outcome. For $2 \leq k \leq m / 2$ we find $k=2$ as the optimal value in case of best or stochastic dominance set comparison, and $k \approx \sqrt{m}$ in case of worst set comparison. For more than two agents it is computationally complex to find the optimal value of $k$, but we conjecture that the optimal $k$ is equal to $\frac{m}{2}$ for a (relatively) large number of agents (see the concluding section).

${ }^{1}$ Maus et al. (2007) contains a brief overview of this literature. 
Of course, counting the non-manipulable profiles is just one way of measuring the degree of (non-)manipulability of voting rules. Many other approaches are possible, see e.g. Campbell and Kelly (2008). Also, our measure of nonmanipulability reflects 'impartial culture': each preference profile is implicitly regarded as equally likely. On the more positive side, approval voting is a well accepted procedure, and our results provide some indication on how to optimize this procedure from the view point of non-manipulability.

The organization of the paper is as follows. Section 2 introduces $k$-approval scoring rules. In Sections 3-5 we determine the non-manipulable preference profiles under the three mentioned preference extensions: worst, best, and stochastic dominance comparison, respectively. Section 6 establishes the optimal (least manipulable) value of $k$ for two agents. Section 7 briefly discusses a variation on worst and best set comparison, namely lexicographic worst and best set comparison. Section 8 concludes.

\section{Approval scoring rules}

The set of agents is $N=\{1, \ldots, n\}$ with $n \geq 2$, and the set of alternatives is $A$ with $|A|=m \geq 3$. (We denote the cardinality of a set $D$ by $|D|$.) A preference is a linear ordering on $A$, i.e., a complete, reflexive, antisymmetric and transitive binary relation on $A$. By $P$ we denote the set of all preferences. A (preference) profile $p$ is a function from $N$ to $P$, i.e., an element of $P^{N}$. For a profile $p$, $p(i)$ is the preference of agent $i \in N$. A social choice correspondence is a map $\varphi: P^{N} \rightarrow 2^{A} \backslash\{\emptyset\}$. Thus, to every profile $p$ the social choice correspondence $\varphi$ assigns a non-empty subset of alternatives $\varphi(p)$.

We next introduce some convenient notation. Let $p \in P^{N}$ and $i \in N$. For a non-empty subset $B$ of $A, p(i)_{\mid B}$ denotes the restriction of $p(i)$ to $B$, hence $p(i)_{\mid B}=\left\{(x, y) \in B^{2} \mid(x, y) \in p(i)\right\}$. For $1 \leq k \leq m$ we denote by $\beta_{k}(p(i))$ the set of the $k$ first ranked alternatives according to $p(i)$, that is, $\beta_{k}(p(i))=\{x \in A||\{y \in A \mid(y, x) \in p(i)\} \mid \leq k\}$. We write $\beta(p(i))$ for the best element of $A$ according to $p(i)$, hence, $\beta_{1}(p(i))=\{\beta(p(i))\}$. Similarly, $\beta\left(p(i)_{\mid B}\right)$ denotes the best element of $B$ according to $p(i)$, that is, $\beta\left(p(i)_{\mid B}\right)=x \in B$ such that $(x, y) \in p(i)$ for all $y \in B$. Also, $\omega\left(p(i)_{\mid B}\right)$ denotes the worst element of $B$ according to $p(i)$, that is, $\omega\left(p(i)_{\mid B}\right)=x \in B$ such that $(y, x) \in p(i)$ for all $y \in B$. The lower contour set of $a \in A$ at $p(i)$ is the set $L(a, p(i))=\{x \in A \mid$ $(a, x) \in p(i)\}$. Observe that $a \in L(a, p(i))$ by reflexivity.

In order to define $k$-approval scoring rules, we denote by the $k$-score

$$
\operatorname{score}_{k}(x, p)=\left|\left\{i \in N \mid x \in \beta_{k}(p(i))\right\}\right|, \quad x \in A, \quad 1 \leq k \leq m, \quad p \in P^{N}
$$

the total number of agents for who alternative $x$ is among the $k$ first ranked alternatives at a profile $p$. The $k$-approval scoring rule $\varphi_{k}$, defined by

$$
\varphi_{k}(p)=\left\{x \in A \mid \operatorname{score}_{k}(x, p) \geq \operatorname{score}_{k}(y, p) \text { for all } y \in A\right\}, \quad p \in P^{N}
$$

assigns to each profile $p$ the subset of alternatives with maximal $k$-score. 
We need a few more notations. For $p \in P^{N}$ and $i \in N, \varphi_{k}\left(p_{-i}\right)$ denotes the set of alternatives assigned by the $k$-approval scoring rule to the restricted profile $p_{-i}=\left(p_{1}, \ldots, p_{i-1}, p_{i+1}, \ldots, p_{n}\right)$, that is,

$$
\varphi_{k}\left(p_{-i}\right)=\left\{x \in A \mid \operatorname{score}_{k}\left(x, p_{-i}\right) \geq \operatorname{score}_{k}\left(y, p_{-i}\right) \text { for all } y \in A\right\}
$$

where $\operatorname{score}_{k}\left(x, p_{-i}\right)=\left|\left\{i \in N \backslash\{i\} \mid x \in \beta_{k}(p(i))\right\}\right|$. Finally, for (any) $a \in$ $\varphi_{k}\left(p_{-i}\right)$,

$$
\varphi_{k}^{-}\left(p_{-i}\right)=\left\{x \in A \mid \operatorname{score}_{k}\left(x, p_{-i}\right)=\operatorname{score}_{k}\left(a, p_{-i}\right)-1\right\}
$$

is the (possibly empty) set of those alternatives that have $k$-score one less than the elements of $\varphi_{k}\left(p_{-i}\right)$. These notations are convenient in view of the following observation, which will be used throughout the paper:

$$
\varphi_{k}(p)=\left\{\begin{array}{cl}
\varphi_{k}\left(p_{-i}\right) \cap \beta_{k}(p(i)) & \text { if } \varphi_{k}\left(p_{-i}\right) \cap \beta_{k}(p(i)) \neq \emptyset \\
\varphi_{k}\left(p_{-i}\right) \cup\left[\varphi_{k}^{-}\left(p_{-i}\right) \cap \beta_{k}(p(i))\right] & \text { if } \varphi_{k}\left(p_{-i}\right) \cap \beta_{k}(p(i))=\emptyset,
\end{array}\right.
$$

for all $p \in P^{N}, i \in N$, and $1 \leq k \leq m$.

In order to define (non-)manipulability of a social choice correspondence at particular preference profiles we need to be able to extend individual preferences to preferences over non-empty subsets of alternatives. For an agent $i$ in $N$ and a preference $p(i) \in P$, we say that a binary relation $\succeq_{p(i)}$ on $2^{A} \backslash\{\emptyset\}$ extends $p(i)$ if $\{x\} \succeq_{p(i)}\{y\} \Leftrightarrow(x, y) \in p(i)$ holds for all $x, y \in A .^{2}$

In this paper we will consider several ways to extend a preference $p(i)$ over alternatives to a binary relation (also called preference) over non-empty sets of alternatives. Suppose that $\succeq_{p(i)}$ extends $p(i)$ for all $i \in N$. For $i \in N$ and $p, q \in P^{N}$, we say that $p$ and $q$ are $i$-deviations if $p_{-i}=q_{-i}$. A choice correspondence $\varphi$ is manipulable by agent $i$ at $p$ towards $q$ if $p$ and $q$ are $i$ deviations and $\varphi(q) \succ_{p(i)} \varphi(p)$, where $\succ_{p(i)}$ is the asymmetric part of $\succeq_{p(i)}$. A social choice correspondence $\varphi$ is not manipulable at $p$ if for all agents $i$ there is no $i$-deviation $q$ such that $\varphi$ is manipulable by $i$ at $p$ towards $q$.

In the following sections we will characterize the profiles at which the $k$ approval scoring rule is not manipulable by any agent, for several different preference extensions.

\section{Non-manipulability under worst comparison}

In this section we extend preferences to sets by considering the worst alternatives of those sets. Let $i \in N$ and $p(i) \in P$, then we define the extension ${ }^{3} \succeq_{p(i)}$ by

$$
B \succeq_{p(i)} C \Leftrightarrow\left(\omega\left(p(i)_{\mid B}\right), \omega\left(p(i)_{\mid C}\right)\right) \in p(i)
$$

\footnotetext{
${ }^{2}$ We write $B \succeq_{p(i)} C$ instead of $(B, C) \in \succeq_{p(i)}$.

${ }^{3}$ In order to avoid cumbersome notation we will use the same symbols for different preference extensions in this paper.
} 
for all non-empty sets $B, C \in 2^{A}$. Thus, $B$ is preferred to $C$ whenever the worst element of $B$ is preferred, according to $p(i)$, to the worst element of $C$.

The following theorem characterizes all profiles at which the $k$-approval scoring rule is not manipulable.

Theorem 3.1 Let $p \in P^{N}$ be a profile. The k-approval scoring rule $\varphi_{k}$ is not manipulable at $p$ if and only if for each agent $i$ at least one of the following three statements holds:

(a) $\left|\varphi_{k}\left(p_{-i}\right) \cap \beta_{k}(p(i))\right|=1$.

(b) $\left|\varphi_{k}\left(p_{-i}\right)\right|=1$.

(c) $A \backslash \beta_{k}(p(i)) \subsetneq \varphi_{k}\left(p_{-i}\right)$.

In words, this theorem says the following. The $k$-approval scoring rule is nonmanipulable at some preference profile if and only if for each agent at least one of the following holds: (a) exactly one of that agent's $k$ first ranked alternatives is among the winners without that agent's vote; (b) without that agent's vote there is a unique winner; (c) all alternatives not ranked among that agent's first $k$ alternatives are winners without that agent's vote, but there are other such winners as well.

Proof of Theorem 3.1. For the if-part, let $i \in N$ and let $q$ be an $i$-deviation of $p$. Note that $\varphi_{k}\left(p_{-i}\right)=\varphi_{k}\left(q_{-i}\right)$ and $\varphi_{k}^{-}\left(p_{-i}\right)=\varphi_{k}^{-}\left(q_{-i}\right)$. Assume that at least one of the cases (a), (b), and (c) holds. We show that agent $i$ cannot manipulate from $p$ to $q$.

In case (a), let $\{x\}=\varphi_{k}\left(p_{-i}\right) \cap \beta_{k}(p(i))$. By (1), $\varphi_{k}(p)=\{x\}$. Again by (1), either $\varphi_{k}(q) \subseteq \varphi_{k}\left(p_{-i}\right)$ or $\varphi_{k}\left(p_{-i}\right) \subseteq \varphi_{k}(q)$. In the first case, if $x \in \varphi_{k}(q)$, then $\varphi_{k}(p)=\{x\} \succeq_{p(i)} \varphi_{k}(q)$; if $x \notin \varphi_{k}(q)$ then $\varphi_{k}(q) \subseteq A \backslash \beta_{k}(p(i))$ so that again $\varphi_{k}(p)=\{x\} \succeq_{p(i)} \varphi_{k}(q)$. In the second case, $\varphi_{k}(p)=\{x\} \subseteq \varphi_{k}(q)$, hence $\varphi_{k}(p) \succeq_{p(i)} \varphi_{k}(q)$.

In case (b), let $\varphi_{k}\left(p_{-i}\right)=\{x\}$ for some alternative $x$. If $x \in \beta_{k}(p(i))$ we are done by case (a). If $x \notin \beta_{k}(p(i))$ then by (1), $\varphi_{k}(p)=\{x\} \cup\left[\varphi_{k}^{-}\left(p_{-i}\right) \cap \beta_{k}(p(i))\right]$ and, thus, $\omega\left(\varphi_{k}(p)\right)=x$. Further, also by $(1), \varphi_{k}(q)=\{x\}$ or $\varphi_{k}(q)=\{x\} \cup$ $\left[\varphi_{k}^{-}\left(p_{-i}\right) \cap \beta_{k}(q(i))\right]$; in both cases, $\left(x, \omega\left(\varphi_{k}(q)\right) \in p(i)\right.$ and, thus, $\varphi_{k}(p) \succeq_{p(i)}$ $\varphi_{k}(q)$.

In case (c), by (1) we have $\varphi_{k}(p)=\varphi_{k}\left(p_{-i}\right) \cap \beta_{k}(p(i))$ and $\varphi_{k}(q)=\varphi_{k}\left(q_{-i}\right) \cap$ $\beta_{k}(q(i))=\varphi_{k}\left(p_{-i}\right) \cap \beta_{k}(q(i))$. If $\beta_{k}(q(i))=\beta_{k}(p(i))$ then $\varphi_{k}(p)=\varphi_{k}(q)$. Otherwise, since $A \backslash \beta_{k}(p(i)) \subsetneq \varphi_{k}\left(p_{-i}\right)$, there is a $y \in\left[A \backslash \beta_{k}(p(i))\right] \cap \varphi_{k}(q)$. Hence, $\varphi_{k}(p) \succeq_{p(i)} \varphi_{k}(q)$.

For the only-if part, suppose that there is an agent $i \in N$ such that none of the three cases (a), (b), and (c) holds. It is sufficient to prove that $\varphi_{k}$ is manipulable at profile $p$ by agent $i$. For this, in turn, it is sufficient to prove that $i$ can manipulate at profile $p$ for the following two cases.

Case (i): $\varphi_{k}\left(p_{-i}\right) \cap \beta_{k}(p(i))=\emptyset$ and $\left|\varphi_{k}\left(p_{-i}\right)\right| \geq 2$. 
Let $b=\beta\left(p(i)_{\mid \varphi_{k}\left(p_{-i}\right)}\right)$. Take $q(i)$ such that the positions in $p(i)$ of $b$ and one of the alternatives in $\beta_{k}(p(i))$ are swapped. Then $\varphi_{k}(q)=\{b\}$ and $\varphi_{k}(q) \succ_{p(i)}$ $\varphi_{k}(p)$, hence agent $i$ can manipulate at profile $p$ towards $q$.

Case (ii): $\left|\varphi_{k}\left(p_{-i}\right) \cap \beta_{k}(p(i))\right| \geq 2$ and $\left[A \backslash \beta_{k}(p(i))\right] \nsubseteq \varphi_{k}\left(p_{-i}\right)$.

Let $w=\omega\left(p(i)_{\mid \varphi_{k}\left(p_{-i}\right) \cap \beta_{k}(p(i))}\right)$ and $y \in A \backslash\left[\beta_{k}(p(i)) \cup \varphi_{k}\left(p_{-i}\right)\right]$. Let $q(i)$ be obtained from $p(i)$ by swapping the positions of the alternatives $w$ and $y$. By (1), $\varphi_{k}(p)=\varphi_{k}\left(p_{-i}\right) \cap \beta_{k}(p(i))$ and $\varphi_{k}(q)=\varphi_{k}\left(p_{-i}\right) \cap \beta_{k}(p(i)) \backslash\{w\}$ it follows that $\varphi_{k}(q) \succ_{p(i)} \varphi_{k}(p)$, proving that $\varphi_{k}$ is manipulable by agent $i$ at profile $p$ towards $q$.

\section{Non-manipulability under best comparison}

In this section we extend preferences to sets by considering the best alternatives of those sets. Let $i \in N$ and $p(i) \in P$, then we define the extension $\succeq_{p(i)}$ by

$$
B \succeq_{p(i)} C \Leftrightarrow\left(\beta\left(p(i)_{\mid B}\right), \beta\left(p(i)_{\mid C}\right)\right) \in p(i)
$$

for all non-empty sets $B, C \in 2^{A}$. Thus, $B$ is preferred to $C$ whenever the best element of $B$ is preferred, according to $p(i)$, to the best element of $C$.

The following theorem characterizes all profiles at which the $k$-approval scoring rule is not manipulable under 'best comparison' of sets.

Theorem 4.1 Let $p \in P^{N}$ be a profile. The $k$-approval scoring rule $\varphi_{k}$ is not manipulable at $p$ if and only if for each agent $i$ at least one of the following three statements holds:

(a) $\left(\beta\left(p(i)_{\mid \varphi_{k}\left(p_{-i}\right)}\right), x\right) \in p(i)$ for all $x \in \varphi_{k}^{-}\left(p_{-i}\right)$.

(b) $\varphi_{k}\left(p_{-i}\right) \cap \beta_{k}(p(i))=\emptyset$ and $\varphi_{k}^{-}\left(p_{-i}\right) \cap \beta_{k}(p(i)) \neq \emptyset$.

(c) $\left|\varphi_{k}\left(p_{-i}\right) \cap \beta_{k}(p(i))\right|>\left|A \backslash\left[\beta_{k}(p(i)) \cup \varphi_{k}\left(p_{-i}\right)\right]\right|$.

In words, this theorem says the following. The $k$-approval scoring rule is nonmanipulable at some preference profile if and only if for each agent at least one of the following holds: (a) the best element of the winners without that agent's vote is preferred by him over all 'almost' winners without his vote; (b) none of the winners without that agent's vote is among his $k$ first ranked alternatives, but some of the 'almost' winners are; (c) the number of the agent's $k$ first ranked alternatives among the winners without his vote is larger than the number of alternatives that are neither among his $k$ first ranked nor among the winners without his vote.

Proof of Theorem 4.1. For the if-part, let $q$ be an $i$-deviation of $p$. Note that $\varphi_{k}\left(p_{-i}\right)=\varphi_{k}\left(q_{-i}\right)$ and $\varphi_{k}^{-}\left(p_{-i}\right)=\varphi_{k}^{-}\left(q_{-i}\right)$. Assume that at least one of the cases (a), (b), and (c) holds. We show that agent $i$ cannot manipulate from $p$ to $q$. 
In case (a), for both cases occurring in (1), we obtain $\beta\left(p(i)_{\mid \varphi_{k}(p)}\right)=$ $\beta\left(p(i)_{\mid \varphi_{k}\left(p_{-i}\right)}\right)$. Since $\beta\left(p(i)_{\mid \varphi_{k}(q)}\right) \in \varphi_{k}\left(p_{-i}\right) \cup \varphi_{k}^{-}\left(p_{-i}\right)$ and by the assumption for case (a), we conclude that $\varphi_{k}(p) \succeq_{p(i)} \varphi_{k}(q)$.

In case (b), again using (1), we have $\varphi_{k}(p)=\varphi_{k}\left(p_{-i}\right) \cup\left[\varphi_{k}^{-}\left(p_{-i}\right) \cap \beta_{k}(p(i))\right]$, hence $\beta\left(p(i)_{\mid \varphi_{k}(p)}\right)=\beta\left(p(i)_{\mid \varphi_{k}^{-}\left(p_{-i}\right) \cap \beta_{k}(p(i))}\right)$; and $\varphi_{k}(q) \in \varphi_{k}\left(p_{-i}\right) \cup\left[\varphi_{k}^{-}\left(p_{-i}\right) \cap\right.$ $\left.\beta_{k}(q(i))\right]$. By the assumptions for this case, $\varphi_{k}(p) \succeq_{p(i)} \varphi_{k}(q)$.

In case (c), it is easy to see that $\left|A \backslash \varphi_{k}\left(p_{-i}\right)\right|<\left|\beta_{k}(p(i))\right|=k=\left|\beta_{k}(q(i))\right|$, hence $\beta_{k}(q(i)) \cap \varphi_{k}\left(p_{-i}\right) \neq \emptyset$. Therefore, by (1) we have $\varphi_{k}(p)=\varphi_{k}\left(p_{-i}\right) \cap$ $\beta_{k}(p(i))$ and $\varphi_{k}(q)=\varphi_{k}\left(p_{-i}\right) \cap \beta_{k}(q(i)) \subseteq \varphi_{k}\left(p_{-i}\right)$. Thus, also in this case $\varphi_{k}(p) \succeq_{p(i)} \varphi_{k}(q)$.

For the only-if part, suppose that there is an agent $i \in N$ such that none of the three cases (a), (b), and (c) holds. It is sufficient to prove that $\varphi_{k}$ is manipulable at profile $p$ by agent $i$. For this, in turn, it is sufficient to prove that $i$ can manipulate at profile $p$ for the following two cases.

Case (i): There is an $x \in \varphi_{k}^{-}\left(p_{-i}\right)$ such that $(x, b) \in p(i)$, where $b=$ $\beta\left(\varphi_{k}\left(p_{-i}\right), p(i)_{\mid \varphi_{k}\left(p_{-i}\right)}\right) ; \varphi_{k}\left(p_{-i}\right) \cap \beta_{k}(p(i)) \neq \emptyset$; and $\left|\varphi_{k}\left(p_{-i}\right) \cap \beta_{k}(p(i))\right| \leq$ $\left|A \backslash\left[\beta_{k}(p(i)) \cup \varphi_{k}\left(p_{-i}\right)\right]\right|$.

For this case, note that $x \in \beta_{k}(p(i))$. By the assumptions for this case we can take a $q(i) \in P$ with $x \in \beta_{k}(q(i))$ and $\varphi_{k}\left(p_{-i}\right) \cap \beta_{k}(q(i))=\emptyset$. Hence, $x \in \varphi_{k}(q) \backslash \varphi_{k}(p)$ and, thus, $\varphi_{k}(q) \succ_{p(i)} \varphi_{k}(p)$. So $i$ can manipulate at profile $p$ towards $q$.

Case (ii): There is an $x \in \varphi_{k}^{-}\left(p_{-i}\right)$ such that $(x, b) \in p(i)$, where $b=$ $\beta\left(p(i)_{\mid \varphi_{k}\left(p_{-i}\right)}\right)$; and $\varphi_{k}^{-}\left(p_{-i}\right) \cap \beta_{k}(p(i))=\emptyset$.

In this case, $\varphi_{k}(p)=\varphi_{k}\left(p_{-i}\right)$. Note that the sets $\beta_{k}(p(i)), \varphi_{k}\left(p_{-i}\right)$, and $\varphi_{k}^{-}\left(p_{-i}\right)$ are pairwise disjoint. So we can take $q(i) \in P$ such that $x \in \beta_{k}(q(i))$ and $\varphi_{k}\left(p_{-i}\right) \cap \beta_{k}(q(i))=\emptyset$. Then $\varphi_{k}(q) \supseteq \varphi_{k}\left(p_{-i}\right) \cup\{x\}$, so $x \in \varphi_{k}(q) \backslash \varphi_{k}(p)$, thus $\varphi_{k}(q) \succ_{p(i)} \varphi_{k}(p)$ and $i$ can manipulate at profile $p$ towards $q$.

\section{Non-manipulability under stochastic domin- ance comparison}

In this section comparisons of sets of alternatives are based on stochastic dominance. To formalize this we need some further notions. Let $u$ be a function from $A$ to $\mathbb{R}$. Then $u$ is said to be a utility function representing preference $p(i)$ of agent $i$, if for all alternatives $x$ and $y$ in $A$

$$
(x, y) \in p(i) \text { if and only if } u(x) \geq u(y) .
$$

Let $B$ and $C$ be two nonempty subsets of alternatives. Agent $i$ is said to prefer $B$ to $C$ according to stochastic dominance at preference $p(i)$, denoted as $B \succeq_{p(i)} C$, if

$$
\sum_{a \in B} \frac{1}{|B|} u(a) \geq \sum_{a \in C} \frac{1}{|C|} u(a) \text { for every utility function } u \text { representing } p(i) \text {. }
$$


This preference extension ${ }^{4}$ is based on the idea that, if we attach equal probabilities to the alternatives in each set, then the expected utility of the resulting lottery over $B$ should be at least as high as the expected utility of the resulting lottery over $C$, for each utility function representing $p(i)$. Clearly, and in contrast to worst and best comparison in the preceding sections, this preference extension is not complete: many sets are incomparable. Observe that our notion of manipulability implies that an agent manages to obtain a preferred and thus comparable set.

In the following theorem we characterize the non-manipulable profiles under the stochastic dominance preference extension. To understand the proof, it is sometimes convenient to keep in mind the familiar characterization (or definition) of stochastic dominance involving only probabilities. This characterization says that a lottery $\ell$ is preferred over another lottery $\ell^{\prime}$ if it can be obtained by shifting probability in $\ell^{\prime}$ to preferred alternatives.

Theorem 5.1 Let $p \in P^{N}$ be a profile. The $k$-approval scoring rule $\varphi_{k}$ is not manipulable at $p$ if and only if for all agents $i$ at least one of the following five statements holds:

(a) $A \backslash \beta_{k}(p(i)) \subsetneq \varphi_{k}\left(p_{-i}\right)$.

(b) $\varphi_{k}\left(p_{-i}\right) \subseteq\left[A \backslash \beta_{k}(p(i))\right]$ and $\varphi_{k}^{-}\left(p_{-i}\right) \cap \beta_{k}(p(i)) \neq \emptyset$.

(c) $\varphi_{k}\left(p_{-i}\right) \cap \beta_{k}(p(i))=\{w\}$ for some $w \in A$ and $\left[A \backslash \beta_{k}(p(i))\right] \cap \varphi_{k}\left(p_{-i}\right) \neq \emptyset$.

(d) $\varphi_{k}\left(p_{-i}\right)=\{w\}$ for some $w \in A$ and $\varphi_{k}^{-}\left(p_{-i}\right) \subseteq L(w, p(i))$.

(e) $\varphi_{k}\left(p_{-i}\right)=\{w\}$ for some $w \in A$ and $\left|\varphi_{k}^{-}\left(p_{-i}\right) \cap L(w, p(i))\right|>m-k$.

In words, these five cases can be described as follows. In case (a), all of agent $i$ 's lower ranked alternatives and at least one of his $k$ first ranked alternatives are among the winners without $i$ 's vote. In case (b), all winners without $i$ 's vote are lower ranked by $i$ but there is an 'almost winner' without $i$ 's vote that is among $i$ 's $k$ first ranked alternatives. In case (c) there is a unique alternative among the winners without $i$ 's vote that is also among his $k$ first ranked alternatives, but there are also alternatives among those winners that are lower ranked by $i$. In case $(\mathrm{d})$ there is a unique winner without $i$ 's vote, and all 'almost' winners without $i$ 's vote are worse for $i$ than this unique alternative. In case (e) there is again a unique winner without $i$ 's vote, and among the 'almost' winners without $i$ 's vote there are more than $m-k$ alternatives worse than this unique alternative.

Proof of Theorem 5.1. For the if-part, let $q$ be an $i$-deviation of $p$. Note that $\varphi_{k}\left(p_{-i}\right)=\varphi_{k}\left(q_{-i}\right)$ and $\varphi_{k}^{-}\left(p_{-i}\right)=\varphi_{k}^{-}\left(q_{-i}\right)$. Assume that at least one of the cases (a)-(e) holds. We show that agent $i$ cannot manipulate from $p$ to $q$.

\footnotetext{
${ }^{4}$ The stochastic dominance criterion to compare sets has been used before, see e.g. Barberà et al. (2001).
} 
In case (a), we have $\varphi_{k}(p)=\varphi_{k}\left(p_{-i}\right) \cap \beta_{k}(p(i))$ and $\varphi_{k}(q)=\varphi_{k}(p)$ or $\varphi_{k}(q) \cap\left[A \backslash \beta_{k}(p(i))\right] \neq \emptyset$. In both cases, it is easy to see that $\varphi_{k}(p) \succeq_{p(i)} \varphi_{k}(q)$.

In case $(\mathrm{b}), \varphi_{k}(p)=\varphi_{k}\left(p_{-i}\right) \cup\left[\varphi_{k}^{-}\left(p_{-i}\right) \cap \beta_{k}(p(i))\right]$. If $\beta_{k}(q(i)) \cap \varphi_{k}\left(p_{-i}\right)=\emptyset$ then $\varphi_{k}(q)=\varphi_{k}\left(p_{-i}\right) \cup\left[\varphi_{k}^{-}\left(p_{-i}\right) \cap \beta_{k}(q(i))\right]$ but this is never preferred to $\varphi_{k}(p)$ according to $\succeq_{p(i)}$. If $\beta_{k}(q(i)) \cap \varphi_{k}\left(p_{-i}\right) \neq \emptyset$ then $\varphi_{k}(q)=\beta_{k}(q(i)) \cap \varphi_{k}\left(p_{-i}\right)$, which again is never preferred to $\varphi_{k}(p)$ according to $\succeq_{p(i)}$.

In case (c), (d), and (e), $\varphi_{k}(p)=\{w\}$. If $\varphi_{k}(q) \neq\{w\}$ then $x \in \varphi_{k}(q)$ for some $x \in L(w, p(i)) \backslash\{w\}$. In that case $\varphi_{k}(q)$ is never preferred to $\varphi_{k}(p)$ according to $\succeq_{p(i)}$.

For the only-if part, suppose that there is an agent $i \in N$ such that none of the five cases (a)-(e) holds. It is sufficient to prove that $\varphi_{k}$ is manipulable at profile $p$ by agent $i$.

By taking negations it follows that none of (a)-(e) holding is equivalent to all of the following four statements holding for $p$ and $i$.

I (i) $A \backslash \beta_{k}(p(i)) \nsubseteq \varphi_{k}\left(p_{-i}\right)$ or (ii) $A \backslash \beta_{k}(p(i))=\varphi_{k}\left(p_{-i}\right)$.

II (i) $\varphi_{k}\left(p_{-i}\right) \nsubseteq\left[A \backslash \beta_{k}(p(i))\right]$ or (ii) $\varphi_{k}^{-}\left(p_{-i}\right) \cap \beta_{k}(p(i))=\emptyset$.

III (i) $\left|\varphi_{k}\left(p_{-i}\right) \cap \beta_{k}(p(i))\right| \geq 2$ or (ii) $\varphi_{k}\left(p_{-i}\right) \cap \beta_{k}(p(i))=\emptyset$ or (iii) $\varphi_{k}\left(p_{-i}\right) \subseteq$ $\beta_{k}(p(i))$.

IV (i) $\left|\varphi_{k}\left(p_{-i}\right)\right|>1$ or (ii) $\left[\varphi_{k}\left(p_{-i}\right)=\{w\}\right.$ for some $w \in A$ and $\varphi_{k}^{-}\left(p_{-i}\right) \nsubseteq$ $L(w, p(i))$ and $\left.\left|\varphi_{k}^{-}\left(p_{-i}\right) \cap L(w, p(i))\right| \leq m-k\right]$.

Requiring (I)-(IV) to hold implies 24 cases to consider. However, the following combinations are contradictory: I(ii) and III(i); I(ii) and III(iii); II(i) and III(ii); and III(i) and IV(ii). Moreover, III(iii) and IV(i) together imply III(i), so that we do not have the case with IV(i) and III(iii) separately. This leaves eight cases, which we will consider two at a time.

A. Cases I(i) \& II(i) \& III(i) \& IV(i) and I(i) \& II(ii) \& III(i) \& IV(i). These two cases are covered by the following assumptions: $\left|\varphi_{k}\left(p_{-i}\right) \cap \beta_{k}(p(i))\right| \geq 2$ and $A \backslash \beta_{k}(p(i)) \nsubseteq \varphi_{k}\left(p_{-i}\right)$.

In this case we have $\varphi_{k}(p)=\varphi_{k}\left(p_{-i}\right) \cap \beta_{k}(p(i))$. Let $w=\omega\left(p(i)_{\mid \varphi_{k}\left(p_{-i}\right) \cap \beta_{k}(p(i))}\right)$. Then we can construct an $i$-deviation $q$ of $p$ such that $\varphi_{k}\left(p_{-i}\right) \cap \beta_{k}(q(i))=\left[\varphi_{k}\left(p_{-i}\right) \cap \beta_{k}(p(i))\right] \backslash\{w\}$. Then $\varphi_{k}(q)=$ $\left[\varphi_{k}\left(p_{-i}\right) \cap \beta_{k}(p(i))\right] \backslash\{w\}$ and, thus, $\varphi_{k}(q) \succeq_{p(i)} \varphi_{k}(p)$.

B. Cases I(i) \& II(ii) \& III(ii) \& IV(i) and I(ii) \& II(ii) \& III(ii) \& IV(i). These two cases are covered by the following assumptions: $\left|\varphi_{k}\left(p_{-i}\right)\right|>1$ and $\varphi_{k}\left(p_{-i}\right) \cap \beta_{k}(p(i))=\emptyset$ and $\varphi_{k}^{-}\left(p_{-i}\right) \cap \beta_{k}(p(i))=\emptyset$.

In this case we have $\varphi_{k}(p)=\varphi_{k}\left(p_{-i}\right)$. Let $b=\beta\left(p(i)_{\mid \varphi_{k}\left(p_{-i}\right)}\right)$. We can construct an $i$-deviation $q$ of $p$ such that $\varphi_{k}\left(p_{-i}\right) \cap \beta_{k}(q(i))=\{b\}$. Then $\varphi_{k}(q)=$ $\{b\} \succ_{p(i)} \varphi_{k}\left(p_{-i}\right)=\varphi_{k}(p)$.

C. Cases I(i) \& II(ii) \& III(ii) \& IV(ii) and I(ii) \& II(ii) \& III(ii) \& IV(ii). These two cases are covered by the following assumptions: $\left[\varphi_{k}\left(p_{-i}\right)=\{w\}\right.$ for 
some $w \in A$ and $\varphi_{k}^{-}\left(p_{-i}\right) \nsubseteq L(w, p(i))$ and $\left.\left|\varphi_{k}^{-}\left(p_{-i}\right) \cap L(w, p(i))\right| \leq m-k\right]$ and $\varphi_{k}\left(p_{-i}\right) \cap \beta_{k}(p(i))=\emptyset$ and $\varphi_{k}^{-}\left(p_{-i}\right) \cap \beta_{k}(p(i))=\emptyset$.

In this case $\varphi_{k}(p)=\{w\}$. Let $b=\beta\left(p(i)_{\mid \varphi_{k}^{-}\left(p_{-i}\right)}\right)$. Then $(b, w) \in p(i)$ since $\varphi_{k}^{-}\left(p_{-i}\right) \not L L(w, p(i))$. Construct an $i$-deviation $q$ of $p$ such that $\varphi_{k}^{-}\left(p_{-i}\right) \cap$ $\beta_{k}(q(i))=\{b\}$. Then $\varphi_{k}(q)=\{b, w\} \succ_{p(i)}\{b\}=\varphi_{k}(p)$.

D. Cases I(i) \& II(i) \& III(iii) \& IV(ii) and I(i) \& II(ii) \& III(iii) \& IV(ii). These cases are covered by the assumptions: $\left[\varphi_{k}\left(p_{-i}\right)=\{w\}\right.$ for some $w \in A$ and $\varphi_{k}^{-}\left(p_{-i}\right) \not L L(w, p(i))$ and $\left.\left|\varphi_{k}^{-}\left(p_{-i}\right) \cap L(w, p(i))\right| \leq m-k\right]$ and $\varphi_{k}\left(p_{-i}\right) \subseteq$ $\beta_{k}(p(i))$.

In this case, $\varphi_{k}(p)=\{w\}$. We can construct an $i$-deviation $q$ of $p$ such that $\varphi_{k}^{-}\left(p_{-i}\right) \cap L(w, p(i)) \subseteq A \backslash \beta_{k}(q(i))$. Then $\varphi_{k}(q)=\{w\} \cup\left[\varphi_{k}^{-}\left(p_{-i}\right) \cap \beta_{k}(q(i))\right]$. The set $\varphi_{k}^{-}\left(p_{-i}\right) \cap \beta_{k}(q(i))$ is non-empty and contains only points $x$ with $(x, w) \in$ $p(i)$. Hence, $\varphi_{k}(q) \succ_{p(i)} \varphi_{k}(p)$.

\section{Minimal manipulability in the two-agent case}

In this section we concentrate on the two-agent case and consider the following question: which $k$-approval scoring rule is the least manipulable, under various assumptions on preference extensions as studied in the preceding sections?

We start with a simple theorem, which will be derived from Theorems 3.1, 4.1 , and 5.1, but also easily follows directly. Call a social choice correspondence strategy-proof if it is not manipulable at any profile $p$.

Theorem 6.1 Let $n=2$. Then the 1-approval scoring rule $\varphi_{1}$ is strategy-proof under worst, best, and stochastic dominance comparison.

Proof. Let $p=(p(1), p(2))$ be a preference profile and let $k=1$. Note that (b) in Theorem 3.1 is always satisfied: this shows strategy-proofness under worst comparison. In Theorem 4.1, (a) reduces to $\beta(p(1))=\beta(p(2))$ and (b) to $\beta(p(1)) \neq \beta(p(2))$ : this shows strategy-proofness under best comparison. Finally, in Theorem 5.1, (b) reduces to $\beta(p(1)) \neq \beta(p(2))$ and (d) to $\beta(p(1))=\beta(p(2))$ : this shows strategy-proofness under stochastic dominance comparison.

This observation might make our quest for minimally manipulable rules futile, were it not the case that the 1-approval scoring rule (i.e., plurality rule) is not unambiguously attractive. As an example, consider the case where agent 1 has preference $p(1): x z \ldots y$ and agent 2 has preference $p(2): y z \ldots x$ (notations obvious). Then $\varphi_{1}(p)=\{x, y\}$ but $\varphi_{2}(p)=\{z\}$. So it seems that $\varphi_{2}$ offers a better compromise in this case than $\varphi_{1}$.

Moreover, for more than two agents and apart from a few particular cases, Theorem 6.1 no longer holds.

We will now consider the three cases (worst, best, and stochastic dominance comparison separately). 


\subsection{Worst comparison for two agents}

The non-manipulable profiles for two agents under worst comparison are easily described using Theorem 3.1.

Corollary 6.2 Let $n=2$ and $2 \leq k<m$. Let $p \in P$ be a profile. Consider worst comparison.

(a) If $k \leq(m+1) / 2$, then $\varphi_{k}$ is not manipulable at $p$ if and only if $\left|\varphi_{k}(p)\right|=1$, or equivalently,

$$
\left|\beta_{k}(p(i)) \cap \beta_{k}(p(2))\right|=1 .
$$

(b) If $k>(m+1) / 2$, then $\varphi_{k}$ is not manipulable at $p$ if and only if $\left|\varphi_{k}(p)\right|=$ $2 k-m$, or equivalently,

$$
\left|\beta_{k}(p(i)) \cap \beta_{k}(p(2))\right|=2 k-m .
$$

Proof. Case (b) in Theorem 3.1 does not apply. If case (a) in Theorem 3.1 applies then we have $\left|\beta_{k}(p(1)) \cap \beta_{k}(p(2))\right|=1$ (or, equivalently, $\left|\varphi_{k}(p)\right|=1$ ), but this is possible if and only if $k \leq(m+1) / 2$. If case (c) in Theorem 3.1 applies then we have $\left|\beta_{k}(p(1)) \cap \beta_{k}(p(2))\right|=2 k-m$ (or, equivalently, $\left|\varphi_{k}(p)\right|=2 k-m$ ), but this is possible if and only if $k \geq(m+1) / 2$; but for $k=(m+1) / 2$ we have $2 k-m=1$, so that we are back in case (a).

Denote by $\eta(m, k)$ the number of profiles (for two agents) at which $\varphi_{k}$ is not manipulable. By straightforward counting we obtain the following result for the number of manipulable profiles for two agents under worst comparison.

Theorem 6.3 Let $n=2$ and $2 \leq k<m$. Consider worst comparison. Then

$$
\eta(m, k)=\left\{\begin{array}{c}
m ! k\left(\begin{array}{c}
m-k \\
k-1
\end{array}\right) k !(m-k) ! \quad \text { if } k \leq(m+1) / 2 \\
m !\left(\begin{array}{c}
k \\
2 k-m
\end{array}\right) k !(m-k) ! \quad \text { if } k>(m+1) / 2 .
\end{array}\right.
$$

Based on this theorem we now show the following facts about the optimal $k$ as far as non-manipulability is concerned:

(i) $\eta(m, k)$ increases in $k$ between 2 and an integer $k^{*}$, which is close to $\sqrt{m}$, and decreases between $k^{*}$ and $\frac{1}{2}(m-1)$.

(ii) $\eta(m, k)$ increases between $\frac{1}{2}(m-1)$ and $(m-1)$.

(iii) The $(m-1)$-approval scoring rule is second best since $\eta(m,(m-1))>$ $\eta(m, k)$ for all $m-1>k \geq 2$.

The first-best value of $k$ is $k=1$ (Theorem 6.1 ), but $\varphi_{1}$ has the drawback that it does not give much opportunity for compromises. Among other values of $k$, the value $k=m-1$ is best. We might, however, prefer to have $k \leq(m+1) / 2$, for the following reason. Call a social choice correspondence citizen-sovereign 
if for every alternative $x \in A$ there is a profile $p \in P$ with $\varphi(p)=\{x\}$. It is not difficult to see that $\varphi_{k}$ is citizen-sovereign for any number of agents $n \geq 2$ if $k \leq(m+1) / 2$. For $n=2$ and $k>(m+1) / 2$, however, $\varphi_{k}$ is not citizensovereign. Hence, if we restrict ourselves to citizen-sovereign rules with $k \geq 2$, then the best rule is $\varphi_{k^{*}}$, where $k^{*}$ is close to $\sqrt{m}$.

We now derive the statements (i), (ii) and (iii) above. For $2 \leq k \leq(m+1) / 2$ we have

$$
\eta(m, k)=\frac{m ! k^{2}[(m-k) !]^{2}}{(m-2 k+1) !}
$$

by Theorem 6.3. By a few elementary computations we obtain for $2 \leq k \leq$ $(m+1) / 2-1$ :

$\eta(m, k)<[>] \eta(m, k+1) \Leftrightarrow(m-k)^{2} k^{2}<[>](k+1)^{2}(m-2 k+1)(m-2 k)$.

Denote $A:=(k+1)^{2}(m-2 k+1)(m-2 k)$, then $A>(k+1)^{2}(m-2 k)^{2}$ and this latter expression at least as large as $(m-k)^{2} k^{2}$ if and only if $k^{2}+2 k-m \leq 0$, which, in turn, holds if and only if $k \leq \sqrt{m+1}-1$. Thus, by $(*)$,

$$
k \leq \underline{k}:=\sqrt{m+1}-1 \Rightarrow \eta(m, k)<\eta(m, k+1) .
$$

Similarly, $A<(k+1)^{2}(m-2 k+1)^{2}$ and this latter expression is not larger than $(m-k)^{2} k^{2}$ if and only if $k^{2}+k-(m+1) \geq 0$, which, in turn, holds if and only if $k \geq \frac{1}{2} \sqrt{1+4(m+1)}-\frac{1}{2}$. Thus, by $(*)$,

$$
k \geq \bar{k}:=\frac{1}{2} \sqrt{1+4(m+1)}-\frac{1}{2} \Rightarrow \eta(m, k)>\eta(m, k+1) .
$$

It is straightforward to derive that $\underline{k}<\bar{k}<(m+1) / 2$ and $\bar{k}-\underline{k}<1$. Now statement (i) follows by taking $k^{*}=\lfloor\underline{k}\rfloor+1$ or $k^{*} \in\{\lfloor\underline{k}\rfloor+1,\lfloor\underline{k}\rfloor+2\}$, depending on the exact values of $k$ and $\bar{k}$.

For $m-1 \geq k>(m+1) / 2$ we have

$$
\eta(m, k)=\frac{m ! k ! k !}{(2 k-m) !}
$$

by Theorem 6.3. To go from $\eta(m, k)$ to $\eta(m, k+1)$ we multiply by a factor $(k+1)^{2} /(2 k-m+2)(2 k-m+1)$, which is larger than 1 since $k+1 \geq 2 k-m+2$. This proves statement (ii) above.

To show statement (iii) we have to show $\alpha(m, k)<1$ for all $2 \leq k \leq$ $(m+1) / 2$, where $\alpha(m, k)=\eta(m, k) / \eta(m, m-1)$. By a simple computation we derive

$$
\alpha(m+1, k)=\alpha(m, k) \cdot \frac{(m+1-k)^{2}}{m^{2}(m-2 k+2)} .
$$

Since $(m+1-k)^{2}<m^{2}$ and $m-2 k+2 \geq 1$, this implies $\alpha(m+1, k)<\alpha(m, k)$. Hence, to show $\alpha(m, k)<1$ for all $2 \leq k \leq(m+1) / 2$, it is sufficient to show $\alpha(2 k+1, k)<1$ for all $k \geq 2$. We show this by induction on $k$. For $k=2$ we have $\alpha(5,2)=3 / 4<1$. Now assume $\alpha(2 k+1, k)<1$ then it is sufficient to 
show $\alpha(2 k+3, k+1)<1$. To show this, by straightforward computation we have

$$
\alpha(2 k+3, k+1)=\alpha(2 k+1, k) \cdot \frac{(k+2)^{2}}{2 k \cdot(2 k+1)} .
$$

By the induction hypothesis and since $(k+2)^{2}<2 k \cdot(2 k+1)$ for $k \geq 2$, we obtain $\alpha(2 k+3, k+1)<1$.

\subsection{Best comparison for two agents}

The non-manipulable profiles for two agents under best comparison can be derived from Theorem 4.1.

Corollary 6.4 Let $n=2$ and $2 \leq k<m$. Let $p \in P$ be a profile. Consider best comparison.

(a) If $k \leq m / 2$ then $\varphi_{k}$ is not manipulable at $p \in P$ if and only if either

$$
\beta(p(1)) \in \beta_{k}(p(2)) \text { and } \beta(p(2)) \in \beta_{k}(p(1))
$$

or

$$
\beta_{k}(p(1)) \cap \beta_{k}(p(2))=\emptyset .
$$

(b) If $k>m / 2$ then $\varphi_{k}$ is not manipulable at any $p \in P$.

Proof. If $k>m / 2$ then case (c) in Theorem 4.1 applies to all $p \in P$, and if $k \leq m / 2$ then case (c) applies to no $p \in P$. This implies part (b) of the corollary, and it also implies that for $k \leq m / 2$ we only have to consider cases (a) and (b) in Theorem 4.1. It is easily seen that these cases result in the two cases in part (a) of the corollary.

The number of non-manipulable profiles $\eta(m, k)$ if $k \leq m / 2$ is computed in the following theorem.

Theorem 6.5 Let $n=2$ and $2 \leq k \leq m / 2$. Consider best comparison. Then

$$
\eta(m, k)=m !(m-2) !(k-1)^{2}+m !(m-1) !+m ![(m-k) !]^{2} /(m-2 k) ! .
$$

Proof. The first case in (a) in Corollary 6.4 with $\beta(p(1)) \neq \beta(p(2))$ results in

$$
m !(k-1)\left(\begin{array}{c}
m-2 \\
k-2
\end{array}\right)(k-1) !(m-k) !
$$

different non-manipulable profiles. This yields the first term of $\eta(m, k)$ in the theorem. If $\beta(p(1))=\beta(p(2))$ then this number is simply equal to $m !(m-1)$ !, which yields the second term. The second case in (a) in Corollary 6.4 results in

$$
m !\left(\begin{array}{c}
m-k \\
k
\end{array}\right) k !(m-k) !
$$


different non-manipulable profiles, which simplifies to the third term for $\eta(m, k)$ in the theorem.

If we require $k \neq 1$ and citizen-sovereignty, i.e., $k \leq m / 2$, then the optimal value of $k$ with respect to non-manipulability, i.e., the value of $k$ that maximizes $\eta(m, k)$, is equal to 2 .

To see this, note that by Theorem 6.5 and some elementary calculations we have for $2<k \leq \frac{m}{2}$ :

$$
\begin{aligned}
& \eta(m, 2)>\eta(m, k) \\
& \Leftrightarrow(m-2)(m-3)>k(k-2)+\overbrace{k-2 \text { factors }}^{\frac{(m-k)(m-k-1) \cdot \ldots \cdot(m-2 k+1)}{\underbrace{(m-2)}_{(m-2)(m-3) \cdot \ldots \cdot(m-k+1)}}} .
\end{aligned}
$$

Since $k>2$ it is therefore sufficient to prove that

$$
(m-2)(m-3)>k(k-2)+(m-2 k+2)(m-2 k+1) .
$$

This simplifies to $(4 k-8) m>5 k^{2}-8 k-4$. Since $m \geq 2 k$, it is sufficient to show that $3 k^{2}-8 k+4>0$, which indeed holds for $k>2$.

\subsection{Stochastic dominance comparison for two agents}

The non-manipulable profiles for two agents under stochastic dominance comparison can be derived from Theorem 5.1.

Corollary 6.6 Let $n=2$ and $2 \leq k<m$. Let $p \in P$ be a profile. Consider stochastic dominance comparison. Then $\varphi_{k}$ is not manipulable at $p$ if and only if at least one of the following holds.

(a) $\beta_{k}(p(1)) \cap \beta_{k}(p(2))=\emptyset$.

(b) $\left|\beta_{k}(p(1)) \cap \beta_{k}(p(2))\right|=1$.

(c) $\beta_{k}(p(1)) \cap \beta_{k}(p(2)) \neq \emptyset$ and $\left[A \backslash \beta_{k}(p(1))\right] \cap\left[A \backslash \beta_{k}(p(2))\right]=\emptyset$.

Proof. For $n=2$ and $k \geq 2$ cases (d) and (e) in Theorem 5.1 are not possible. Case (c) in Theorem 5.1 reduces to case (b) above, and case (a) in Theorem 5.1 reduces to case (c) above. Finally, case (b) in the theorem reduces to case (a) above.

From this description we can again derive the number of manipulable profiles $\eta(m, k)$.

Theorem 6.7 Let $n=2, k \geq 2$, and consider stochastic dominance comparison.

(a) If $k \leq m / 2$ then

$$
\eta(m, k)=m ![(m-k) !]^{2} /(m-2 k) !+m ! k^{2}[(m-k) !]^{2} /(m-2 k+1) ! .
$$


(b) If $k>m / 2$ then

$$
\eta(m, k)=m ![k !]^{2} /(2 k-m) ! .
$$

Proof. If $k \leq m / 2$ then (c) in Corollary 6.6 is not possible, and cases (a) and (b) in the corollary are mutually exclusive. In case (a) of Corollary 6.6 there are

$$
m !\left(\begin{array}{c}
m-k \\
k
\end{array}\right) k !(m-k) !
$$

non-manipulable profiles, resulting in the first term for $\eta(m, k)$, and in case (b) of the corollary there are

$$
m ! k\left(\begin{array}{c}
m-k \\
k-1
\end{array}\right) k !(m-k) !
$$

non-manipulable profiles, resulting in the second term for $\eta(m, k)$.

If $k>m / 2$ then case (a) of Corollary 6.6 is not possible, and (b) is a special case of (c). For the latter case, we just have to count the number of profiles for which $\left[A \backslash \beta_{k}(p(1))\right] \cap\left[A \backslash \beta_{k}(p(2))\right]=\emptyset$, since the other condition is always fulfilled. This number is equal to

$$
m !\left(\begin{array}{c}
k \\
m-k
\end{array}\right) k !(m-k) !
$$

which is equal to $m ![k !]^{2} /(2 k-m) !$.

About the value of $k$ that maximizes $\eta(m, k)$, so the value of $k$ that is optimal with respect to non-manipulability, we can say the following.

1. For $2 \leq k \leq \frac{m}{2}$, the number of non-manipulable profiles decreases with $k$, and thus $k=2$ is optimal.

2. For $\frac{m}{2}<k \leq m-1$, the number of non-manipulable profiles increases with $k$, and thus $k=m-1$ is optimal.

3. $\eta(m, 2)>\eta(m, m-1)$ for $m \geq 4$, so $k=2$ is the overall optimal value between 2 and $m-1$.

To prove these statements, first assume $k \leq \frac{m}{2}$. Then, using Theorem 6.7(a) and simplifying, we derive

$$
\eta(m, k+1)<\eta(m, k) \Leftrightarrow 3 k^{2}-2 k m-1<0,
$$

and it is easily seen that the right hand side holds for all $2 \leq k \leq \frac{m}{2}$. Next, assume $\frac{m}{2}<k \leq m-1$. Then, using Theorem 6.7(b) and simplifying, we derive

$$
\eta(m, k+1)>\eta(m, k) \Leftrightarrow 3 k^{2}+k(4-4 m)+m^{2}-3 m+1<0 .
$$

The roots of the quadratic expression in $k$ at the right hand side are $\frac{2}{3}(m-$ 1) $\pm \frac{1}{3} \sqrt{m^{2}+m+1}$; the smaller root is smaller than $\frac{m}{2}$, whereas the larger root 
is larger than $m-1$. Thus, the right hand side holds for all $\frac{m}{2}<k \leq m-1$. Finally, by Theorem 6.7 again,

$$
\eta(m, 2)>\eta(m, m-1) \Leftrightarrow m>3,
$$

so that $k=2$ is the overall optimal value of $k$ for $2 \leq k \leq m-1$.

\section{Lexicographic worst and best comparison}

In this section we briefly consider a natural extension of worst and best comparison, namely lexicographic worst and best comparison. These preference extensions to sets are given by the following recursive definition. For two subsets $B$ and $C$ of alternatives, we say that $B$ is (weakly) preferred to $C$ under lexicographic worst comparison by agent $i$ with preference $p(i)$ if

1. $C=\emptyset$, or

2. $B$ and $C$ are non-empty and $\left(\omega\left(p(i)_{\mid B}\right), \omega\left(p(i)_{\mid C}\right)\right) \in p(i)$, or

3. $\omega\left(p(i)_{\mid B}\right)=\omega\left(p(i)_{\mid C}\right)=: w$ and $B \backslash\{w\}$ is preferred to $C \backslash\{w\}$ under lexicographic worst comparison by agent $i$ with preference $p(i)$.

The definition for lexicographic best comparison is obtained simply by replacing the worst alternative by the best alternative, i.e., by replacing $\omega(\cdot)$ by $\beta(\cdot)$. Thus, under lexicographic worst comparison an agent first considers the worst elements of $B$ and $C$. If these are different, then he prefers the set with the better worst element. Otherwise, the agent considers the second worst elements. If these are different, then he prefers the set with the better second worst element. Otherwise, he considers the third worst elements, etc. Similarly, of course, for lexicographic best comparison.

Complete characterizations of the non-manipulable profiles for both lexicographic worst and lexicographic best comparison can be given but are rather technical (even more so than for stochastic dominance comparison) and therefore not included in this version.

Note that any profile that is manipulable under worst [best] comparison is also manipulable under lexicographic worst [best] comparison. Hence, the set of non-manipulable profiles under lexicographic worst [best] comparison is always a subset of the set of non-manipulable profiles under worst [best] comparison. It is not very difficult to check (we omit the proof for the sake of briefness) that all the profiles listed in Corollary 6.2, that is, all two-agent profiles that are non-manipulable under worst comparison, are also non-manipulable under lexicographic worst comparison, so that in this case considering lexicographic worst comparison instead of just worst comparison does not make any difference. The non-manipulable profiles coincide, and the optimal value of $k$ as far as nonmanipulability is concerned, is the same as in Section 6.1.

For two agents and lexicographic best comparison the situation is different and the set of non-manipulable profiles is a strict subset of the set of nonmanipulable profiles under best comparison, that is, the set of profiles described 
in Corollary 6.4. To be precise, we have the following result which can be derived from Corollary 6.4 (the proof is again left to the reader).

Corollary 7.1 Let $n=2$ and $2 \leq k<m$. Let $p \in P$ be a profile. Consider lexicographic best comparison.

(a) If $k \leq m / 2$ then $\varphi_{k}$ is not manipulable at $p \in P$ if and only if either

$$
\{\beta(p(1))\}=\{\beta(p(2))\}=\beta_{k}(p(1)) \cap \beta_{k}(p(1))
$$

or

$$
\beta_{k}(p(1)) \cap \beta_{k}(p(2))=\emptyset .
$$

(b) If $k>m / 2$ then $\varphi_{k}$ is not manipulable at any $p \in P$.

In this case, the total number of non-manipulable profiles for $2 \leq k \leq m / 2$ is equal to

$$
\eta(m, k)=\frac{m ![(m-k) !]^{2}(m-2 k+2)}{(m-2 k+1) !}
$$

and this number is decreasing in $k$, so that $k=2$ is the value of $k$ that minimizes manipulability subject to $2 \leq k \leq m / 2$, just as in the best comparison case. The proofs of these facts are somewhat simpler than for the best comparison case. For the sake of briefness we omit them.

\section{Concluding remarks}

In this paper we have characterized the number of non-manipulable preference profiles under $k$-approval voting for several ways of comparing sets, i.e., by considering worst or best alternatives, or by applying a stochastic dominance criterion. We have found the optimal $k$ - the maximal number of non-manipulable profiles - for the case of two agents.

If there is a very large number of agents (for instance, elections for national Parliaments), then it is very unlikely that a single agent will have any influence at all, whatever voting procedure is used. In that case the non-manipulability issue becomes irrelevant, at least as manipulation by single agents is concerned.

It is interesting to consider situations where the number of agents is relatively large compared to the number of alternatives, but not so large that individual influences are nihil (for instance, elections of officers or council members of scientific communities). We conjecture that in such a case choosing $k$ equal to $m / 2$ is optimal from the point of view of non-manipulability, not only within the class of $k$-approval rules but even within the class of all scoring rules. The intuition is that, statistically, the variance in scores is maximal for this value of $k$, so that any single agent's probability of being able to change the outcome is minimal. 


\section{References}

Barberà S, Dutta B, Sen A (2001) Strategy-proof social choice correspondences. Journal of Economic Theory 101:374-394

Brams SJ, Fishburn PC (1983) Approval voting. Birkhauser, Boston MA

Campbell DE, Kelly JS (2008) Gains from manipulation of social choice rules. Economic Theory (forthcoming)

Gibbard A (1973) Manipulation of voting schemes: a general result. Econometrica 41: 587-602

Kelly JS (1988) Minimal manipulability and local strategy-proofness. Social Choice and Welfare 5: 81-85

Kelly JS (1989) Interjacency. Social Choice and Welfare 6: 331-335

Maus S, Peters H, Storcken T (2007) Minimal manipulability: unanimity and nondictatorship. Journal of Mathematical Economics 43:675-691.

Peters H, Roy S, Storcken T (2009) Manipulability of approval voting. Mimeo, University of Maastricht

Satterthwaite M (1975) Strategy-proofness and Arrow's conditions: existence and correspondence theorems for voting procedures and social welfare functions. Journal of Economic Theory 10: 187-217 